\title{
Motor and Occipital Cortex Excitability in Migraine Patients
}

\author{
Sefer Gunaydin, Aysun Soysal, Turan Atay, Baki Arpaci
}

\begin{abstract}
Objective: We evaluated motor and occipital cortex excitability in migraine patients using transcranial magnetic stimulation. Methods: In this study, we included 15 migraine patients with aura (MwA), 15 patients without aura (MwoA) between attacks, and 31 normal healthy controls. Motor thresholds at rest, amplitudes of motor evoked potentials, central motor conduction time and cortical silent period were measured by stimulation of the motor cortex by using $13.5 \mathrm{~cm}$ circular coil and recording from abductor digiti minimi muscle. Additionally, phosphene production and the threshold of phosphene production was determined by stimulation of the visual cortex with the same coil. Results: No significant differences were observed between the groups with respect to the motor thresholds, Motor evoked potential max/compound muscle action potential max (MEPmax/Mmax) amplitudes, central motor conduction times and duration of cortical silent period. Although not statistically significant, the proportion of the migraineurs with phosphene generation (90\%) was found to be higher than that of normal controls $(71 \%)$. Phosphene threshold levels in migraine patients, however, were significantly lower than those of the controls with MwA patients having the lowest levels. Conclusion: Our findings indicate that the occipital cortex, but not the motor cortex, is hyperexcitable in migraine patients.
\end{abstract}

RÉSUMÉ: Excitabilité du cortex moteur et du cortex occipital chez les patients migraineux. Objectif: Nous avons évalué par stimulation magnétique transcrânienne l'excitabilité du cortex moteur et du cortex occipital chez des patients migraineux. Méthodes: Nous avons étudié entre les crises 15 patients ayant une migraine avec aura (MaA), 15 patients migraineux sans aura (MsA) et 31 témoins en bonne santé. Les seuils moteurs au repos, les amplitudes des potentiels évoqués moteurs (PÉM), le temps de conduction moteur central et la période de silence cortical ont été mesurés par stimulation du cortex moteur au moyen d'une spirale circulaire de 13,5 cm et enregistrement au niveau du muscle abducteur du petit doigt. De plus, la production de phosphènes et le seuil de production de phosphènes ont été déterminés par stimulation du cortex visuel avec la même spirale. Résultats: Nous n'avons observé aucune différence significative entre les groupes quant aux seuils moteurs, aux amplitudes PÉMmax/Mmax, aux temps de conduction motrice centrale et à la durée des périodes de silence cortical. Bien que ce ne soit pas significatif au point de vue statistique, la proportion de migraineux qui ont eu des phosphènes $(90 \%)$ était plus élevée que celle des témoins normaux $(71 \%)$. Le niveau seuil de phosphènes chez les migraineux était cependant significativement plus bas que celui des témoins et les MaA avaient le seuil le plus bas. Conclusion: Nos observations indiquent que le cortex occipital est hyperexcitable chez les migraineux, mais que le cortex moteur ne l'est pas.

Can. J. Neurol. Sci. 2006; 33: 63-67

Recent studies have demonstrated that central neuronal hyperexcitability has an important role in migraine pathogenesis. Hyperexcitability of occipital cortex has been proposed to be responsible for the elaboration of migraine attacks and visual aura. Motor symptoms in some migraine subtypes have also drawn attention to the hyperexcitability of motor cortex..$^{1-20}$

Transcranial magnetic stimulation (TMS) is a non-invasive tool to investigate physiology and excitability of both motor- and occipital cortices. ${ }^{11,21-25}$ The changes in motor threshold, latency and amplitude of motor evoked potential, central motor conduction time and cortical silent period after magnetic stimulation of the corticospinal tract can be used to identify quantitative evidence of motor cortex excitability. ${ }^{3-4}$ Similarly, phosphene generation and the threshold of phosphene induction

From the Neurology Department, Bakirkoy State Hospital for Psychiatric and Neurological Diseases, Bakirkoy, Istanbul, Turkey.

ReCEIVEd February 15, 2005. ACCEPTED In FinAl fORM SEPTEMBer 10, 2005. Reprint requests to: Aysun Soysal, Atakoy 5. Kisim E1/1A Blok Daire: 8 34158 Bakirkoy/Istanbul, Turkey. 
after visual cortex stimulation gives opportunity to assess the excitability of the occipital cortex. . $^{5,6}$

Limitations in previous studies have included small numbers of patients, stimulation of only motor or occipital cortex and other technical issues. In this study, we aimed to investigate the excitability of both motor- and occipital cortices in migraine patients with (MwA)- and without aura (MwoA) by using TMS and to compare the results with those of healthy controls.

\section{Methods}

\section{Subjects}

Fifteen patients with MwA (14 females, one male; mean age: 33.9 \pm 5.9 years; range: $26-46)$ and 15 patients with MwoA (12 females, three males; mean age: $33.0 \pm 4.3$; range: $27-45)$ were compared to 30 healthy controls (26 females, four males; mean age: $33.0 \pm 4.9$; range: $26-45$ ) gathered from members of the hospital staff. Migraine diagnosis was established according to the criteria of the International Headache Society. ${ }^{26}$ The duration of disease was $11.6 \pm 8.2$ and $8.2 \pm 6.4$ years, the attack frequency was $2.6 \pm 1.6$ and $3.5 \pm 1.7$ per month, the duration of the pain was $28.4 \pm 12.2$ and $25.2 \pm 14.3$ hours per attack, the verbal analog scale (from 0 to 4 ) was $3.5 \pm 0.5$ and $3.4 \pm 0.6$, and the visual analog scale (from 0 to 10 ) was $8.67 \pm 1.0$ and $8.53 \pm 1.3$ respectively in MwA and MwoA patients.

All subjects had normal neurological examination before and at the time of the study. The study was performed in the interictal period with a headache-free period of at least one week prior to the study. All migraine patients had no attacks for the three days after the recording. None of the patients was taking prophylactic therapy, nor they were using any drug that could be responsible for changing central nervous system excitability, such as sedatives, hypnotics, anticonvulsants or beta blockers. The patients were allowed to take analgesics if necessary, for a period of six weeks except 48 hours before the study. The examiners were blinded to whether the patients had aura during the attacks or not. The study protocol was approved by the local ethical committee and a written consent form was obtained from all participants.

\section{Experimental Procedures and Recordings}

Subjects were seated in a semi-darkened, silent room. Magnetic stimulations were performed with a Magstim200 stimulator using a $135 \mathrm{~mm}$ circular coil. The coil was centered at the vertex. The direction of the current was clockwise to stimulate the right hemisphere, and anticlockwise to stimulate the left hemisphere. Motor evoked potential (MEP) responses were acquired with a Medelec Saphire 4ME device. Electromyogram (EMG) recordings were made from right- and left abductor digiti minimi (ADM) muscle. The active electrode was placed on the muscle belly and the reference on the fifth metacarpophalangeal joint. Firstly, the ulnar nerve was stimulated at the wrist on both sides using an electrical stimulator and the amplitude of the resulting compound muscle action potential (CMAP) was measured. The motor threshold was defined as the stimulus intensity at which a peak-to-peak MEP amplitude of $50 \mu \mathrm{V}$ was obtained in at least three of six consecutive trials. For MEP studies, $120 \%$ of the motor threshold was used as the stimulus intensity. The response with shortest latency and highest amplitude obtained during six consecutive stimuli at maximal stimulator output on both sides at rest was considered as MEPmax. In order to avoid possible amplitude differences between sides due to asymmetrical positioning of the electrodes, the MEPmax/CMAPmax ratio was calculated. For calculating central motor conduction time (CMCT), the latency of MEP elicited by stimulation over the spinous process of the 7 th cervical vertebra was subtracted from the cortical latency. ${ }^{21-25}$

The cortical silent period (CSP) was studied by stimulating the left motor cortex along with recordings from the right ADM muscle during slight contraction of approximately $30 \%$ maximum voluntary contraction as determined by audio-visual feedback. Maximum stimulus intensity of the stimulator was used. A series of six stimuli was given and the shortest CSP value was selected for evaluation.

During the occipital cortex study, subjects were seated in the same room with eyes closed (wearing a black mask) and the center of the magnetic coil was held against the occipital scalp in the midline, $7 \mathrm{~cm}$ superior to the inion. To avoid bias, subjects were not informed about expectations but asked to report all sensations they experienced. Stimulus intensity was increased by steps of $10 \%$ until a visual phenomena was obtained or the $100 \%$ stimulus intensity was reached. As soon as a phosphene production (bright scintillations in the subject's visual field) was reported by the subjects, stimulus intensity was fine-tuned to determine the thresholds for phosphenes. In patients without phosphene production at the $100 \%$ level of stimulation, the stimulator was moved in $1 \mathrm{~cm}$ steps in all directions, and the same procedure was repeated as mentioned above.

\section{Statistics}

Analysis of the demographic data in migraine patients and their comparisons with controls was performed by using Student's t-test, chi -square test and Mann Whitney-U test. Oneway analysis of variance was used to search for comparisons between MwA patients, MwoA patients and controls. Post hoc analysis was used for comparisons between Mwa/MwoA, MwA/controls and MwoA/controls. Both Spearman's and Pearson's correlation tests were used for correlations.

\section{RESULTS}

There was no significant difference between the mean age of MwA, MwoA patients and controls. Duration of the disease, attack frequency, attack duration, verbal and visual analog scale was not found to be significantly different in MwA and MwoA patients.

No significant differences between groups were found in respect to the right- and left motor threshold, cortical MEP latencies and amplitudes, MEPmax/CMAPmax ratio and CMCT. There were also no statistically significant inter-side differences in any measurements. The duration of the CSP was also similar in three groups (Table I).

The phosphene production of all the migraine patients $(90 \%)$ compared with the controls (71\%), had no significant difference. During occipital cortex stimulation, $93.3 \%$ of the MwA patients, $86.7 \%$ of MwoA patients and $71 \%$ of controls reported phosphenes and there was also no difference between the groups. The mean threshold for eliciting phosphenes was significantly 
Table 1: MEP and CSP values after motor cortex stimulation

\begin{tabular}{|c|c|c|c|c|}
\hline & $\begin{array}{c}\text { MwA } \\
(\operatorname{mean} \pm S D)\end{array}$ & $\begin{array}{c}\text { MwoA } \\
(\text { mean } \pm S D)\end{array}$ & $\begin{array}{c}\text { Controls } \\
(\text { mean } \pm S D)\end{array}$ & $\mathrm{P}$ \\
\hline RMT & $36.2 \pm 6.1$ & $38.7 \pm 3.6$ & $37.2 \pm 4.9$ & 0.403 \\
\hline LMT & $36.8 \pm 6.5$ & $39.3 \pm 4.7$ & $37.7 \pm 5.4$ & 0.466 \\
\hline R cortical MEP amp & $3.2 \pm 0.8$ & $3.3 \pm 1.1$ & $3.0 \pm 0.9$ & 0.544 \\
\hline L cortical MEP amp & $3.2 \pm 1.2$ & $3.1 \pm 1.2$ & $2.9 \pm 0.8$ & 0.684 \\
\hline R MEPmax/CMAPmax & $0.27 \pm 0.07$ & $0.26 \pm 0.09$ & $0.27 \pm 0.08$ & 0.994 \\
\hline L MEPmax/CMAPmax & $0.27 \pm 0.09$ & $0.26 \pm 0.1$ & $0.27 \pm 0.08$ & 0.905 \\
\hline R cortical MEP latency & $20.0 \pm 1.1$ & $19.8 \pm 1.3$ & $20.4 \pm 1.2$ & 0.366 \\
\hline L cortical MEP latency & $20.0 \pm 0.8$ & $19.8 \pm 1.3$ & $20.1 \pm 1.1$ & 0.883 \\
\hline $\mathrm{R}$ CMCT & $7.8 \pm 0.5$ & $7.7 \pm 0.8$ & $7.7 \pm 0.7$ & 0.729 \\
\hline L CMCT & $7.9 \pm 0.5$ & $7.7 \pm 0.9$ & $7.7 \pm 0.7$ & 0.544 \\
\hline R CSP & $189.1 \pm 27.8$ & $197.3 \pm 28.9$ & $189.0 \pm 26.5$ & 0.599 \\
\hline
\end{tabular}

$\mathrm{R}=$ Right; $\mathrm{L}=\mathrm{Left} ; \mathrm{MT}=$ motor threshold; $\mathrm{MEP}=$ motor evoked potential; $\mathrm{CMAP}=$ compound muscle action potential; $\mathrm{CMCT}=$ central motor conduction time; $\mathrm{CSP}=$ cortical silent period.

lower in migraine patients $(46.2 \pm 18.8)$ than that of controls (72.5 $\pm 12.4 ; \mathrm{p}<0.001)$. MwA patients $(35.6 \pm 11.9)$, MwoA patients $(57.8 \pm 18.2)$ and controls $(72.5 \pm 12.4)$ compared together the mean threshold of phosphene production was found significantly different $(\mathrm{p}<0.001)$. By using post hoc analysis test, we found that the threshold of phosphene production was lower in MwA patients than in both MwoA patients and controls $(\mathrm{p}<$ 0.001 and $p<0.001)$. Similarly, it was lower in MwoA patients than in controls (p: 0.008).

Motor threshold, MEP amplitude, CSP and the mean threshold of phosphene production was not correlated with disease duration, attack frequency, attack duration, verbal and visual analog scale $(r<0.25$ for all parameters).

\section{Discussion}

Migraine pathogenesis is not completely understood. It has been speculated that increased cortical excitability might be responsible. Several factors such as a deficit in mitochondrial oxidative phosphorylation, calcium-channel dysfunction, intracellular magnesium deficiency or a combination thereof may lead to neuronal hyperexcitability. ${ }^{11,27-33}$ Transcranial magnetic stimulation is a noninvasive method to assess cortical hyperexcitability. ${ }^{11,21-25}$

The results of TMS studies in migraine patients are controversial. There are studies supporting the presence of cortical hypoexcitability represented by higher motor threshold values. ${ }^{1,2,5}$ On the other hand, van der Kamp et $a^{3,4}$ reported increased MEP/CMAP amplitude ratios and MEP amplitudes indicating a motor cortex hyperexcitability in migraine patients. Another two studies, however, showed no interictal changes of excitability of the motor cortex in migraine. ${ }^{10,16}$

In our study performed during the interictal period, we did not find any significant difference in either MwA and MwoA patients compared with controls, in respect to the motor threshold, cortical MEP latencies and amplitudes, MEPmax/CMAPmax ratio and CMCT. Similarly, no interhemispheric difference between these parameters was noted. Our results support the studies $^{10,16}$ indicating that there is no hyperexcitability of motor cortex in migraine patients during interictal period.

Cortical silent periods are induced by stimulation of the motor cortex and represent an inhibitory phenomenon. ${ }^{24,25}$ It has been postulated that hyperexcitability of the motor cortex observed in some migraine patients might partly be explained by an impairment of inhibitory mechanisms. Therefore, CSP studies have also been performed in migraine patients but the results are controversial.

Such controversial results reported may be due to methodological differences (recordings during slight contraction or at rest, using different stimulus intensity etc.). Three different studies showed that the duration of CSP in migraine patients did not significantly differ from that of controls., ${ }^{5,10,16}$ On the other hand, using low intensity magnetic stimulation, Aurora et $\mathrm{al}^{8}$ found shortened CSP in MwA patients; but at maximal intensity levels of stimuli, this difference disappeared. In the light of these findings they suggested that there might be a dysfunction of GABAergic inhibitory interneurons depending on the level of stimulus intensity. There are many studies in the literature reporting abnormalities observed only at high- or low stimulus intensities. Additionally, paired pulse paradigms are needed to examine different inhibitory and excitatory circuits in the motor cortex. Our results support three prior studies showing no difference in CSP durations between MwA and MwoA patients and controls. However, it should be taken into consideration that neither low stimulus intensities nor paired pulse paradigms were used in this study. Therefore, we might have missed some possible changes by using only maximum stimulus intensity.

The hyperexcitability of the occipital cortex has been considered as the basis for spontaneous or triggered visual aura. ${ }^{6,9}$ Transcranial magnetic stimulation, is therefore, another useful and non-invasive method to assess the hyperexcitability of

Table 2: The prevalence and thresholds of phosphene production after occipital cortex stimulation

\begin{tabular}{l|c|c|c|c}
\hline & \multicolumn{1}{c}{ MwA } & MwoA & Controls & P \\
\hline $\begin{array}{l}\text { Prevalance of } \\
\text { phosphene production }\end{array}$ & $93.3 \%$ & $86.7 \%$ & $71 \%$ & 0.157 \\
$\begin{array}{l}\text { Thresholds for } \\
\text { phosphene production } \\
\text { (mean } \pm \text { SD) }\end{array}$ & $35.6 \pm 11.9$ & $57.8 \pm 18.2$ & $72.5 \pm 12.4$ & $<0.001$ \\
\hline
\end{tabular}


the occipital cortex by eliciting the perception of phosphenes. ${ }^{6,9,11,34}$ The threshold for phosphene production can be used for evaluating visual cortex excitability. $6,9,11,34$

To our knowledge, most TMS studies, with exception of a few, in the literature, showed that migraine patients, especially those with aura, had higher prevalence and lower threshold for phosphene production. ${ }^{5,35}$ In three different studies, Aurora et $\mathrm{al}^{6,7,9,18}$ found higher prevalence and lower threshold of phosphene elicitation in MwA or in both MwA and MwoA patients, respectively. They suggested that these findings could be considered as a direct neurophysiologic evidence for enhanced hyperexcitability of the occipital cortex in migraine patients.

Mulleners et $\mathrm{al}^{11}$ found no significant difference in prevalence of phosphenes but lower did find a threshold of phosphene induction in both MwA and MwoA patients than controls. In their following study, they showed that both MwA- and MwoA patients had lower thresholds than normal controls for phosphene induction, but there was no significant difference in terms of phosphene prevalence between the groups. Although a significant reduction in headache frequency was observed, change in phosphene threshold among migraine patients was highly variable after valproate therapy. ${ }^{15}$ Delivering TMS over visual area V5, Battelli et al ${ }^{13}$ demonstrated that the threshold for phosphene induction was lower in migraine patients and thus, hyperexcitability of the visual cortex extended beyond visual area V1. Using low frequency repetitive TMS (rTMS), Brighina et $\mathrm{al}^{14}$ also reported hyperexcitability of visual cortex in migraineurs. In another study with rTMS in MwA patients, a failure of inhibitory circuits also involving the extrastriate cortex was reported. ${ }^{19}$

A study by Afra et $\mathrm{al}^{5}$ showing occipital hypoexcitability rather than hyperexcitability in MwA patients was later criticized by Aurora and $\mathrm{Welch}^{36}$ In their letter they suggested that this controversy might be explained by different methods used in different studies (i.e. type of stimulator, size of coil, intensity of the stimulus). Similarly, Bohotin et $\mathrm{al}^{35}$ found higher phosphene threshold in both MwA and MwoA patients, and considered this finding as evidence of hypoexcitability of the visual cortex during the interictal period. A different (eight shaped) stimulator they used may have played a role in these results.

Although not statistically significant, the prevalence of phosphene production was found to be higher in both Mwa and MwoA in our study. The size of coil we used may have led to this result. On the other hand, the threshold of phosphene production was significantly lower in MwA patients than those of both MwoA patients and controls. Our results are in concordance with other studies suggesting an occipital cortex hyperexcitabilityrather than motor cortex hyperexcitability in migraine patients.

\section{REFERENCES}

1. Betucci D, Cantello R, Gianelli M, Naldi P, Mutani R. Menstrüel migraine without aura: cortical excitability to magnetic stimulation. Headache. 1992; 32: 345-7.

2. Maertens de Noordhout A, Pepin JL, Schoenen J, Delwaide PJ. Percutaneous magnetic stimulation of the motor cortex in migraine. Electroencephalogr Clin Neurophysiol. 1992; 85: 110-5.

3. van der Kamp W, Maassen VanDenBrink A, Ferrari MD, van Dijk JG. Interictal cortical hyperexcitability in migraine patients demonstrated with transcranial magnetic stimulation. J Neurol Sci. 1996; 139: 106-10.
4. van der Kamp W, Maassen Van Den Brink A, Ferrari MD, van Dijk JG. Interictal cortical excitability to magnetic stimulation in familial hemiplegic migraine. Neurology. 1997; 48: 1462-4.

5. Afra J, Mascia A, Gerard P, de Noordhout MA, Schoenen J. Interictal cortical excitability in migraine: a study using transcranial magnetic stimulation of motor and visual cortices. Ann Neurol. 1998; 44: 209-15.

6. Aurora SK, Welch KMA. Brain excitability in migraine: evidence from transcranial magnetic stimulation studies. Curr Opin Neurol. 1998; 11: 205-9.

7. Aurora SK, Ahmad BK, Welch KMA, Bhardwaj P, Ramadan NM. Transcranial magnetic stimulation confirms hyperexcitability of occipital cortex in migraine. Neurology. 1998; 50: 1111-4.

8. Aurora SK, Al-Sayeed F, Welch KMA. The cortical silent period is shortened in migraine with aura. Cephalalgia. 1999; 19: 708-12.

9. Aurora SK, Cao Y, Bowyer SM, Welch KMA. The occipital cortex is hyperexcitable in migraine: experimental evidence. Headache. 1999; 39: 469-76.

10. Werhahn KJ, Wisenen K, Herzog J, et al. Motor cortex excitability in patients with migraine with aura and hemiplegic migraine. Cephalalgia. 2000; 20: 45-50.

11. Mulleners WM, Chronicle EP, Palmer JE, Koehler PJ, Vredeveld JW. Visual cortex excitability in migraine with and without aura. Headache. 2001;41: 565-72.

12. Mulleners WM, Chronicle EP, Palmer JE, Koehler PJ, Vredeveld JW. Supression of perception in migraine: evidence for reduced inhibition in the visual cortex. Neurology. 2001; 56: 178-83.

13. Battelli L, Black KR, Wray SH. Transcranial magnetic stimulation of visual area V5 in migraine. Neurology. 2002; 58: 1066-9.

14. Brighina F, Piazza A, Daniele O, Fierro B. Modulation of visual cortical excitability in migraine with aura: effects of $1 \mathrm{~Hz}$ repetetive transcranial magnetic stimulation. Exp Brain Res. 2002; $145: 177-81$.

15. Mulleners WM, Chronicle EP, Vredeveld JW, Koehler PJ. Visual cortex excitability in migraine before and after valproate prophylaxis: a pilot study using TMS. Eur J Neurol. 2002; 9: 3540.

16. Ozturk V, Cakmur R, Donmez B, et al. Comparison of cortical excitability in chronic migraine (transformed migraine) and migraine without aura. J Neurol. 2002; 249: 1268-71.

17. Ambrosini A, de Noordhout AM, Sandor PS, Schoenen J. Electrophysiological studies in migraine: a comprehensive review of their interest and limitations. Cephalalgia. 2003; 23 Suppl 1: 13-31.

18. Aurora SK, Welch KMA, Al-Sayeed F. The threshold for phosphenes is lower in migraine. Cephalalgia. 2003; 23: 258-63.

19. Fierro B, Ricci R, Piazza A, et al. $1 \mathrm{~Hz}$ rTMS enhances extrastriate cortex activity in migraine: evidence of a reduced inhibition? Neurology. 2003; 61: 1446-8.

20. Fumal A, Bohotin V, Vandenheede M, Schoenen J. Transcranial magnetic stimulation in migraine: a review of facts and controversies. Acta Neurol Belg. 2003; 103: 144-54.

21. Rossini PM. The anatomic and physiologic bases of motor evoked potentials. In: Gilmore R, editor. Neurologic clinics: evoked potentials. Philadelphia: W.B. Saunders Company;1988. p. 75169.

22. Kimura J. Somatosensory and motor evoked potentials. In: Kimura J, editor. Electrodiagnosis in diseases of nerve and muscle: principles and practice. Philadelphia: F.A. Davis Company; 1989. p. 375-426.

23. Murray NMF. Motor evoked potentials. In: Aminoff MJ, editor. Electrodiagnosis in clinical neurology. New York: Churchill Livingstone; 1992. p. 605-26.

24. Cros D, Chiappa KH. Motor evoked potentials. In: Chiappa KH, editor. Evoked potentials in clinical medicine. Philadelphia: Lippincott-Raven Publishers; 1997. p. 477-507.

25. Robinson LR. Magnetic stimulation of the central and peripheral nervous systems. In: Dumitri D, Amato AA, Zwarts MJ, editors. Electrodiagnostic medicine. Philadelphia: Hanley\&Belfus, inc; 2002. p. 415-27. 
26. Headache Classification Committee of the International Headache Society. Classification and diagnostic criteria for headache disorders, cranial neuralgies and facial pain. Cephalalgia. 1988; 8 Suppl 7: 1-96.

27. Ramadan NM, Halvarson H, Vande-Linde A, et al. Low brain magnesium in migraine. Headache. 1989; 29: 590-3.

28. Gallai V, Sarchielli P, Coata G, et al. Serum and salivary magnesium levels in migraine. Results in a group of juvenile patients. Headache. 1992; 32: 132-5.

29. Mauskop A, Altura BT, Cracco RQ, Altura BM. Deficiency in serum ionized magnesium but not total magnesium in patients with migraines. Possible role of $\mathrm{ICa}^{2}+/ \mathrm{IMg}^{2}+$ ratio. Headache. 1993; 33: $135-8$.

30. Aloisi P, Marrelli A, Porto C, Tozzi E, Cerone G. Visual evoked potentials and serum magnesium levels in juvenile migraine patients. Headache. 1997; 37: 383-5.

31. Silberstein SD, Lipton RB, Dalessio DJ. The pathophysiology of primary headache. In: Silberstein SD, Lipton RB, Dalessio DJ, editors. Headache in clinical practice. Oxford: ISIS Medical Media; 1998. p. 41-58.
32. Swanson JW, Dodick DW, Capobianco DJ. Headache and other craniofacial pain. In: Bradley WG, Daroff RB, Fenichel GM, Marsden CD, editors. Neurology in clinical practice. Vol 2. Boston: Butterworth-Heinemann; 2000. p. 1829-78.

33. Ferrari MD, Haan J. Genetics of headache. In: Silberstein SD, Lipton RB, Dalessio DJ, editors. Wolff's headeache and other head pain. Oxford: Oxford University Press; 2001. p. 73-84.

34. Boroojerdi B, Prager A, Muellbacher W, Cohen L.G. Reduction of human visual cortex excitability using $1 \mathrm{~Hz}$. transcranial magnetic stimulation. Neurology. 2000; 54: 1529-31.

35. Bohotin V, Fumal A, Vandenheede M, Bohotin C, Schoenen J. Excitability of visual V1-V2 and motor cortices to single transcranial magnetic stimuli in migraine: a reappraisal using a figure-of-eight coil. Cephalalgia. 2003; 23: 264-70.

36. Aurora SK, Welch KMA. Phosphene generation in migraine (letter). Ann Neurol. 1999; 45: 416-7. 